\title{
Successful transvenous extraction of an endocardial lead more than 35 years old using mechanical systems
}

\author{
Bezpieczne usunięcie ponad 35-letniej elektrody endokawitarnej \\ z użyciem systemów mechanicznych \\ Andrzej Ząbek ${ }^{1}$, Krzysztof Boczar ${ }^{1}$, Maciej Dębski ${ }^{1}$, Jacek Lelakowski ${ }^{1,2}$, Barbara Małecka ${ }^{1,2}$ \\ 'Department of Electrocardiology, John Paul II Hospital in Krakow, Krakow, Poland \\ ${ }^{2}$ Institute of Cardiology, Jagiellonian University Medical College, Krakow, Poland
}

We present a case of a 59-year-old female patient, pacemaker-dependent, who had been diagnosed with third-degree atrioventricular block with syncopes and underwent VVI pacemaker implantation in 1979. A passive fixation, unipolar, ventricular lead Tesla LES 165 was inserted via cephalic vein, placed in the right ventricular apex, and connected to a $\mathrm{CPI}$ pacemaker. After 10 years, during generator change the lead required repair with an $\mathrm{A} 1 \mathrm{~N}$ connector. At the time of second generator replacement insulation failure was diagnosed, which resulted in pacing and sensing disturbances and muscle stimulation. The old ventricular lead was abandoned and a new DDD pacing system was implanted on the ipsilateral side. On the second postoperative day the patient presented with a syncope. Atrial lead dislodgement, acute rise of ventricular pacing threshold, and small localised pericardial effusion without cardiac tamponade were documented (Fig. 1A). In computed tomography the cardiac perforation with pacemaker leads was ruled out. The patient was then transferred to the reference centre in the field of transvenous lead extraction (TLE), where she approved of the decision to extract all the dysfunctional leads and implant a new DDD pacing system. The procedure was conducted in a hybrid operating room with continuous transoesophageal echocardiography monitoring. The new leads were explanted using simple traction. In order to extract the 35-year-old lead we used mechanical systems. First, to increase the stiffness of the lead a Lead Locking Device (Spectranetics) was deployed and locked along the entire lead lumen. Then green and white Byrd dilator sheaths (Cook Vascular Inc.) were used to free the lead from adhesions (Fig. 1B, C). After successful extraction of the leads the new DDD pacing system (MR Conditional) was implanted via the recaptured venous access (Fig. 1D). The procedure was uneventful. During a follow-up period of 16 months a dual-chamber system maintained proper function. The presented case highlights the problem of abandonment of non-functional endocardial leads, which is especially important in young patients. A long dwell time of inactive leads in the cardiovascular system predisposes to development of late complications of permanent pacing. Abandonment of a non-functional lead in a patient may lead to device-related infection and increases the risk of potential future extraction. Additionally, abandoned leads remain the contraindication to magnetic resonance imaging (MRI) studies. After careful comparison of the risk of lead abandonment versus risk of TLE and taking into consideration the good general condition and relatively young age of our patient we decided to extract the abandoned, very old lead despite the class IIb, C indication according to current Heart Rhythm Society (HRS) expert consensus statement on lead extraction. The additional advantage of our choice is MRI compatibility.
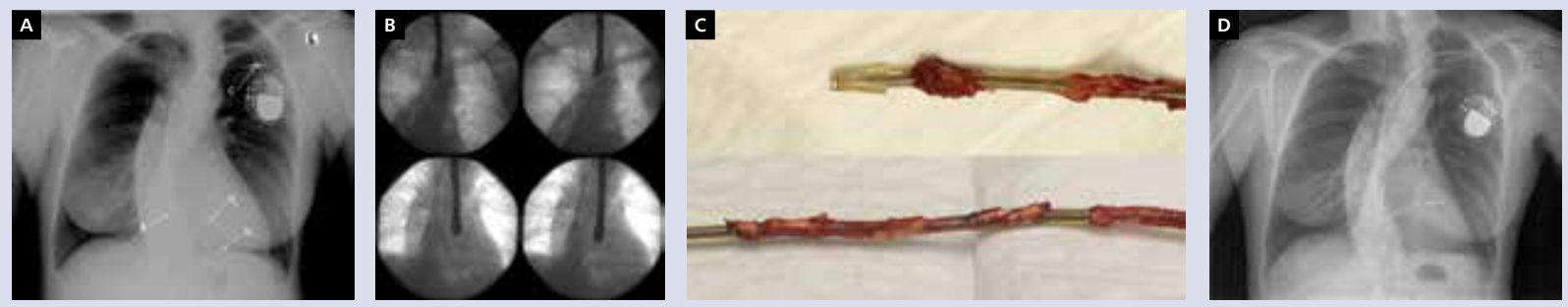

Figure 1. A. Posteroanterior chest $X$-ray before TLE procedure. A - tip of new atrial lead, $V_{1}$ - tip of old ventricular lead, $\mathrm{V}_{2}$ - tip of new ventricular lead, C - A1N connector; B. Subsequent stages of extraction of old ventricular lead. The arrows point to the tip of the Byrd dilator; C. Picture of a transvenously extracted lead over 35 years old. There are visible massive calcified adhesions on the lead; D. Posteroanterior chest X-ray after transvenous lead extraction procedure

Address for correspondence:

Andrzej Ząbek, MD, PhD, Msc, Department of Electrocardiology, John Paul II Hospital in Krakow, ul. Prądnicka 80, 31-202 Kraków, Poland,

e-mail: andrzej__z@poczta.onet.pl

Conflict of interest: none declared

Kardiologia Polska Copyright (c) Polskie Towarzystwo Kardiologiczne 2016 